\title{
CALIDAD DE VIDA RELACIONADA CON LA SALUD EN ADULTOS DIAGNOSTICADOS CON EPILEPSIA
}

\author{
Stefano Vinaccia, Japcy M. Quiceno, Ana M. GaViria y María L. Dey Garzón \\ Universidad de San Buenaventura, Medellín, Colombia
}

\begin{abstract}
Resumen: La presente investigación tuvo como objetivo evaluar la calidad de vida relacionada con la salud en 60 pacientes adultos de ambos géneros con diagnóstico de Epilepsia de la ciudad de Medellín, Colombia. El diseño del estudio fue exploratorio-descriptivo-transversal. Se utilizó el cuestionario específico INCAVI-72 que mide Calidad de Vida Relacionada con la Salud para pacientes con Epilepsia. Los resultados evidenciaron una calidad de vida relacionada con la salud dentro de los límites normales en las dimensiones de Estado de salud y actividades diarias, Satisfacción, Apoyo Social, Efectos adversos de los medicamentos, Impacto de la epilepsia y puntuaciones bajas en las dimensiones de Evaluación de sentimientos y Control del cuestionario INCAVI-72. El alfa de Cronbach del instrumento INCAVI-72 fue de 0,70. En conclusión, el estado emocional y el control personal son factores relevantes en la calidad de vida de los pacientes con epilepsia.
\end{abstract}

Palabras clave: calidad de vida relacionada con la salud, epilepsia, enfermedad crónica, cuestionario INCAVI-72.

\section{Health-Related Quality of Life in adult diagnosed with Epilepsy}

\begin{abstract}
The present research aims at evaluating the health-related quality of life in sixty adult patients of both genders with a diagnosis of Epilepsy, in the city of Medellín, Colombia. The study design was exploratory-descriptive-transversal. The specific questionnaire INCAVI-72 that measures health-related quality of life for patients with Epilepsy, was used. The results demonstrate a health-related quality of life within normal limits of the subscales of: state of health and daily activities, satisfaction, social support, adverse effects of medication, impact of the epilepsy, and with low scores in the subscales of: evaluation of feelings, and control of the INCAVI-72 questionnaire. Cronbach's alfa of the questionnaire INCAVI-72 was found to be 0,70 . In conclusion, the emotional state and the personal control are relevant factors in the health-related quality of life of the patients with epilepsy.
\end{abstract}

Keywords: quality of life, health, epilepsy, chronic disease, INCAVI-72.

\section{INTRODUCCIÓN}

En el campo de la Neurología la Epilepsia es una de los trastornos más prevalentes y se constituye como la segunda gran enfermedad neurológica (Forsgren, 1997; Adams, 1997; Bender, Álvarez y Rodríguez, 2006). Puede presentarse en cualquier edad y a cualquier grupo étnico siendo los infantes y los mayores de 60 años la población más vulnerable (Takeuchi y Gue-

Recibido 20 noviembre 2007; aceptado 18 mayo 2008

Correspondencia: Stefano Vinaccia, Grupo de Investigación Salud Comportamental, Universidad de San Buenaventura de Medellín, Colombia. Carrera 56C, No. 51-90, Apartado Aéreo 81240.Correos-e: vinalpi47@hotmail.com, japcyps@, hotmail.com vara, 1999; Bender, et al., 2006). Este trastorno tiene serias repercusiones no sólo físicas sino también psicosociales que alteran la calidad de vida relacionada con la salud de quienes la padecen (Berrío, 2002).

Históricamente las primeras referencias transcritas están en las tablas cuneiformes neo-babilónicas del Sakikku (718-612 a.C) y etimológicamente el término proviene del griego y significa sorpresa, ataque o caerse sobre sí mismo. Los antiguos griegos a su vez la consideraban una enfermedad divina o sagrada, y más adelante Hipócrates (450 a.C) y Galeno (100 d.C.) fueron quienes le dieron un enfoque más neurológico, describiendo que los ataques provenían del cerebro (García-Albea, 1999; Berrío, 2002). 
En el siglo XIX, el neurólogo John Hughlings Jackson propone lo que podría considerarse la primera teoría neuronal de la epilepsia, y afirma que la «epilepsia es el nombre que se le da a las descargas locales, rápidas, excesivas, súbitas y ocasionales de la sustancia gris» (GarcíaAlbea, 1999). El diccionario de la Organización Mundial de la Salud (OMS) la define como la «Afección crónica producida por diferentes etiologías, caracterizada por la repetición de crisis debidas a una descarga excesiva de las neuronas cerebrales (crisis epilépticas) asociadas eventualmente a síntomas clínicos o paraclínicos» y la Comisión de Epidemiología y Pronóstico de la Liga Internacional contra la Epilepsia (ILAE) la define como la "Condición caracterizada por crisis epilépticas recurrentes (dos o más) no provocadas por alguna causa inmediatamente identificable» (Días, 2002).

\section{PREVALENCIA}

Se considera que más de 50 millones de personas en el mundo padecen de epilepsia y el $80 \%$ viven en países en desarrollo. Quinientos millones de personas tienen un vínculo cercano con las personas afectadas como: familiares, amigos o colegas. El tratamiento farmacológico ha estado disponible alrededor de 90 años y el $75 \%$ de las personas epilépticas son tratadas médicamente y pueden llevar una vida relativamente normal, pero de otro lado, existe un porcentaje significativo de personas que aún no reciben tratamiento médico. Mas sin embargo, otros investigadores consideran que solo el 5\% de estos pacientes reciben el tratamiento adecuado. Algunos expertos en el tema denominan esta situación social como la «paradoja en epilepsia» (Burneo, Tellez-Zenteno y Wiebe, 2005; Bender, Morales, Paz y Prida, 2005; Carrizosa, 2007).

Vélez y Eslava-Cobos (2006) en Colombia realizaron un estudio nacional epidemiológico de las enfermedades neurológicas (EPINEU$\mathrm{RO})$ y plantearon que la prevalencia de la epilepsia en Colombia es similar a la informada en otros países con un desarrollo comparable. La prevalencia general estuvo en 11.3 por 1,000 , con poca variación entre sus diferentes regio- nes. La mayoría de las convulsiones fueron parciales, frecuentemente con generalización secundaria. El síndrome epiléptico más frecuentemente encontrado fue el parcialmente sintomático/criptogénico (80\%). El comienzo de la epilepsia en Colombia es más frecuente en la infancia.

\section{FACTORES DE RIESGO}

La epilepsia es un trastorno con muchas causas posibles. Cualquier circunstancia que obstaculice o distorsione el patrón de actividad neuronal normal puede conducir a la aparición de una crisis epiléptica. A algunas personas epilépticas al tener una cantidad más alta de neurotransmisores activos (sustancias encargadas de conducir el impulso nervioso entre las neuronas) se les incrementa la actividad neuronal, mientras que una baja cantidad de inhibidores de dichos neurotransmisores también pueden llevar al aumento de actividad neuronal. En ambas situaciones aparece la epilepsia (Jiménez, 2004).

Las crisis epilépticas producen una alteración momentánea del funcionamiento cerebral, debida a la descarga súbita y desproporcionada de los impulsos eléctricos que habitualmente utilizan las células del cerebro. Esta descarga puede afectar únicamente a una parte del cerebro (crisis parciales o focales) o comprometer a todo el cerebro (crisis generalizadas) u originarse simultáneamente en ambas áreas hemisféricas cerebrales (crisis parciales secundariamente generalizadas). La epilepsia es regularmente idiopática, lo cual significa que no puede identificarse causa específica para los síntomas (Taylor, 2007). Los síntomas sean éstos físicos o psicológicos que presente una persona durante una crisis epiléptica dependerán de la o las zonas del cerebro que estén siendo afectadas por la descarga (Jiménez, 2004), y las diferentes manifestaciones de las crisis epilépticas le dan el carácter de enfermedad crónica con mala calidad de vida (Herranz, 1999; Bender, et. al., 2005; Bender, et al., 2006) que altera de manera significativa los procesos emotivo-cognitivoconductuales e incluso la personalidad de quienes la sufren, lo que conduce a su vez a un alto 
impacto y costo en el bienestar general de estas personas incluso estando libres de crisis durante muchos años. Sin embargo, según Herranz (1999) no se produce en las personas con epilepsia, como en la mayor parte de las enfermedades crónicas, una relación directa obligada entre la frecuencia y la intensidad de los síntomas físicos y la calidad de vida de las personas que la padecen.

A nivel general, las enfermedades crónicas suelen afectar de manera importante varios aspectos de la vida de los pacientes (Oblitas, 2006), desde la fase inicial aguda, en la que los pacientes sufren un periodo de crisis caracterizado por un desequilibrio físico, social y psicológico, acompañado de ansiedad, depresión, estrés, miedo y desorientación (Brannon y Feist, 2001), hasta la asunción del carácter crónico del trastorno, que implica en mayor o menor medida, cambios permanentes en la actividad física, laboral y social de los pacientes, condición que suele durar toda la vida afectado la calidad de vida (Simón, 1999; Oliveros-Cid, Oliveros-Juste y Cid, 2005; Padilla, 2005; Taylor, 2007).

\section{CVRS EN EPILEPSIA}

Desde el punto de vista subjetivo, la calidad de vida relacionada con la salud (CVRS) es la valoración que realiza una persona, de acuerdo con sus propios criterios del estado físico, emocional y social en que se encuentra en un momento dado, y refleja el grado de satisfacción con una situación personal a nivel: fisiológico (sintomatología general, discapacidad funcional, situación analítica, sueño, respuesta sexual), emocional (sentimientos de tristeza, miedo, inseguridad, frustración), y social (situación laboral o escolar, interacciones sociales en general, relaciones familiares, amistades, nivel económico, participación en la comunidad, actividades de ocio, entre otras) (Schwartzmann, 2003).

En los últimos 25 años se le ha prestado gran atención a la CVRS de los pacientes con enfermedades crónicas. En un principio se consideraba el impacto de los factores físicos y el tratamiento de la enfermedad (Taylor, 2007), pero más adelante se comenzaron a considerar factores psicosociales y psicoemocionales (Oliveros-Cid, et. al., 2005) incluso hasta variables relacionadas con la espiritualidad (Morales, 2002; Padilla, 2005; Giovagnoli, Meneses y Da Silva, 2006). Por lo tanto, a la par del desarrollo del concepto de CVRS se han diseñado instrumentos generales como el cuestionario de salud MOS SF-36 y el cuestionario de calidad de vida diseñado por la OMS WHOQOL 100, los cuales han sido ampliamente utilizados en estudios con pacientes con epilepsia (Giovagnoli, et al., 2006; Laccheo, et al., 2008). Y, para tener una aproximación más detallada tanto médica como psico-social y emocional del impacto de CVRS en los pacientes con epilepsia se han utilizado cuestionarios específicos (véase la Tabla 1).

Los aspectos más significativos que afectan o vulneran la CVRS en los pacientes con epilepsia informados en la literatura en relación con la frecuencia de las crisis han sido los siguientes: A nivel cognitivo-emocional: ansiedad, depresión, estrés, preocupaciones y temo-

Tabla 1. Instrumentos específicos para medir CVRS en adultos con Epilepsia

\section{Instrumentos Especificos}

El Washington Psychosocial Seizure Inventory (WPSI)

Cuestionario de Liverpool

Epilepsy Surgery Inventory (ESI)-55

Quality-of-Life in Epilepsy Inventory QOLIE-89

Quality-of-Life in Epilepsy Inventory QOLIE-10

Escala de Calidad de Vida FEGEA

Spanish Version of the Quality-of-Life in Epilepsy Inventory QOLIE-31

INCAVI-72
Autores

Dodrill, Batzel, Queisser y Temkin (1980)
Baker, et al. (1991)
Vickrey, et al. (1992)
Vickrey, et al. (1993)
Cramer, et al. (1996)
Oliveros (1997)
Torres, et al. (1999)
Berrío, et al. (1999) y Berrío (2002)


res asociados a futuros traumatismos, a las crisis, a la familia, a tener hijos con malformaciones, a las relaciones sexuales y al futuro, $y$ alteraciones generales de las funciones cognitivas. A nivel psicosocial y afectivo: estigma social y discriminación, actividades recreativas (viajar) y las actividades sociales con amigos. A nivel de autoesquemas: autoestima y autoeficacia. A nivel de personalidad: dificultad para sentirse sí mismos. A nivel de actividades de la vida diaria: conducir, independencia, empleo, actividades académicas y domésticas e higiene personal. A nivel del Tratamiento médico: obsesión por el tratamiento médico, preocupación por los efectos secundarios de la medicación y el costo de la enfermedad, y malestar personal por la dependencia a los médicos y entidades hospitalarias. A nivel físico: somnolencia-falta de energía y trastornos del sueño (Oliveros-Cid, et al., 2005).

\section{ANTECEDENTES EMPÍRICOS DE CVRS EN EPILEPSIA}

A nivel general no son muy numerosos los estudios sobre CVRS con muestras de pacientes epilépticos y estos se encuentran desarrollados en diferentes partes del mundo. Las investigaciones de CVRS en epilepsia se han centrado a evaluar por un lado los índices de CVRS y por otro las relaciones entre las emociones negativas como ansiedad y más concretamente la depresión sobre la CVRS (Gil-Nagel y Marinas, 2006).

Cramer, Blum, Reed y Fanning (2003) desarrollaron un estudio en Estados Unidos con el objetivo de evaluar el impacto de la depresión y las crisis epilépticas sobre la CVRS en personas con diagnóstico de epilepsia a través del cuestionario Quality-of-Life in Epilepsy Inventory QOLIE-89 enviado a los 501 participantes del estudio vía correo postal. Los resultados demostraron niveles de CVRS bajos debido a los altos niveles de depresión en todos los tipos de crisis epilépticas. Las personas con predominio mayor, parcial y menor de crisis epilépticas tuvieron significativamente una peor CVRS si tenían depresión mayor o moderada. Por consiguiente, independientemente del tipo de crisis reciente, las personas tuvieron una CVRS considerablemente más baja en todas las dimensiones a comparación de las personas que en los últimos tres meses no habían tenido crisis. En cuanto a diferencias entre grupos en relación a los niveles de depresión encontrados fueron clínicamente significativos: la depresión, las crisis y su severidad y los días de incapacidad con crisis tuvieron una correlación positiva con una baja CVRS en todas las dimensiones a comparación de los pacientes epilépticos que no tenían depresión. Los autores concluyeron que la depresión clínica está significativamente asociada con una baja CVRS independientemente del tipo de crisis epiléptica.

Ríos y Hernández (2005) realizaron un estudio con 205 pacientes con diagnóstico de epilepsia en el Hospital Central Militar de México. El objetivo del estudio fue evaluar la prevalencia de la depresión y la calidad de vida. Como instrumentos se emplearon dos escalas de depresión (Montgomery-Asberg y ZungConde) y una de calidad de vida. Los resultados indicaron alta prevalencia de depresión y una desfavorable calidad de vida en los pacientes que tenían depresión a diferencia de quienes no la presentaron. Por lo tanto, los autores concluyeron que la depresión esta asociada con una baja calidad de vida en los pacientes epilépticos.

Mosaku, Fatove, Komolafe, Lawal y Ola (2006) realizaron un estudio en Nigeria con el objetivo de identificar los factores asociados con la CVRS en pacientes adultos con epilepsia. El estudio estuvo conformado por una muestra de 51 pacientes con epilepsia quienes respondieron cuatro cuestionarios: Quality of Life in Epilepsy Inventory - QOLIE-10, the General Health Questionnaire (GHQ-30), the modified Mini Mental State Examination (mMMSE), y el Hospital Anxiety and Depression Scale (HADS). Los resultados indicaron altos niveles de ansiedad y depresión en los pacientes, siendo la depresión el factor más importante para explicar la baja calidad de vida de éstos. Las otras variables asociadas en una baja CVRS fueron los puntajes del GHQ-30, el género femenino, y la frecuencia de las crisis. Los investigadores concluyeron que un mejor 
control de las crisis epilépticas y una mejor atención a las necesidades psicológicas de los epilépticos adultos tendrían un efecto positivo sobre la CVRS.

Laccheo, et al. (2008) desarrollaron una investigación en los Estados Unidos con el objetivo de evaluar la CVRS en adultos mayores con epilepsia. En el estudio participaron 56 personas mayores de 60 años a quienes se les aplicaron dos instrumentos: uno general (The Quality of Life in Epilepsy Inventory QOLIE-31) y uno especifico (The Short Form 36 - SF-36). Los resultados evidenciaron que las dimensiones de CVRS del instrumento general (SF-36) eran muy inferiores en los pacientes epilépticos en comparación con la población general sana de la misma de edad, hallándose en los primeros limitaciones significativas en las dimensiones emocionales y físicas. En cuanto a los resultados encontrados con el instrumento específico (QOLIE-31) los puntajes fueron similares a otros estudios con muestras poblaciones de la misma edad, encontrándose que el $74 \%$ de los pacientes estaban preocupados sobre los efectos secundarios a largo plazo del tratamiento médico antiepiléptico; el 61\% informaron como significativos los efectos secundarios físicos de la medicación, y el 65\% consideraron los efectos secundarios mentales de la medicación. Los autores concluyeron que existen múltiples factores que pueden afectar la CVRS de las poblaciones de adultos mayores con epilepsia como: variables asociadas al proceso de envejeciendo (demencia, traumatismos, etc.), condiciones de comorbilidad con otros trastornos (depresión), y variables relacionadas específicamente con la epilepsia (independencia, efectos secundarios de la medicación, imprevisibilidad de las crisis, etc.).

Por su parte, Huang, Xie, Li y Wei (2007) en China también desarrollaron un estudio con el objetivo de evaluar la calidad de vida en pacientes con epilepsia. Participaron en el estudio 78 pacientes de epilepsia y 60 controles sanos, a quienes se les aplicó The Quality of Life in Epilepsy Inventory - QOLIE-31. Los resultados demostraron una CVRS muy inferior en comparación con el grupo de control y no hubo diferencias en cuanto al género. De otro lado, se encontró que los pacientes con crisis tónicoclónicas tenían puntajes más altos en la CVRS y más duración en el tiempo entre crisis independientemente del tratamiento médico a diferencia de otros tipos de crisis. Los autores concluyen que la medicación y el tipo de crisis afectan la CVRS en pacientes con la epilepsia.

Guekht, et al. (2007) en Rusia realizaron un estudio con el objetivo de evaluar los diferentes factores sobre salud y la relación con la CVRS en adultos con epilepsia. Participaron en este estudio 242 pacientes de los cuales 214 tenían diagnóstico del síndrome epiléptico parcialmente sintomático/criptogénico y 28 con diagnóstico de epilepsia generalizada idiopática. Se les aplicó The Quality of Life in Epilepsy Inventory - QOLIE-31. Los resultados indicaron que la CVRS general de los pacientes epilépticos fue bastante baja. Se encontró que la frecuencia de las crisis tenía correlaciones muy significativas respecto a la CVRS, igualmente el tiempo de diagnóstico de la enfermedad. Los puntajes más bajos encontrados en el QOLIE31 en relación al tiempo de diagnóstico fue en las subescalas de: energía/fatiga, efectos de la medicación, funcionamiento social, y en la puntuación global del QOLIE-31. Los investigadores concluyeron que la frecuencia de las crisis es el factor más importante sobre la CVRS en adultos con epilepsia recién diagnosticada y previamente tratada.

A partir de lo anterior se planteó como objetivo general de esta investigación evaluar la calidad de vida relacionada con la salud (CVRS) en 60 pacientes adultos de ambos géneros con diagnóstico de Epilepsia de la ciudad de Medellín, Colombia.

\section{MÉTODO}

\section{Participantes}

Se realizó un muestreo por conveniencia noaleatorio, seleccionando a todos los sujetos disponibles de la muestra accesibles. En el estudio participaron voluntariamente 60 personas adultas de ambos géneros con diagnóstico de epilepsia según los criterios de la ILAE (1989) quienes estaban en tratamiento neurológico en 
el Instituto Neurológico de Antioquia de la ciudad de Medellín, Colombia. El rango de edad estuvo comprendido entre los 18 a 55 años con una media de edad de $30,8(D T=8,2)$, siendo los adultos jóvenes de entre 21 a 39 años la muestra poblacional con mayor prevalencia de epilepsia. Sobresalió en el estudio el sexo femenino, 35 mujeres representaron el 58,3\% de la muestra. En relación al estrato socioeconómico colombiano un porcentaje mayor de la muestra $(85 \%)$ se agrupa en el nivel medio-bajo (estratos 2 y 3 ). En cuanto al nivel educativo la mayoría de los participantes $(35 \%)$ no culminaron sus estudios en básica secundaria y sólo un $31,7 \%$ la terminan, un 33,3\% realizaron estudios superiores. A nivel de la variable ocupación, se observó que la mayoría realizan una actividad laboral $(58,3 \%)$ y el resto se ubicó en las categorías ama de casa $(23,3 \%)$ y desempleado (18,3\%). Respecto al estado civil la mitad de la muestra $(51,7 \%)$ esta ubicada en la categoría de solteros, mientras que un $40 \%$ de la muestra se ubica en la categoría casados y unión libre, y un 8,3\% se encontraron separados. Como variable de inclusión se tuvo en cuenta el nivel de escolaridad, preferiblemente personas con estudios mínimos en básica secundaria y que no presentaran deterioro cognitivo.

\section{Instrumentos}

Para evaluar la CVRS se utilizó el Inventario para la evaluación de la Calidad de Vida en adultos con epilepsia INCAVI-72, diseñado por Berrío, et al. (1999; 2002) para adultos mayores de 18 años con diagnóstico de epilepsia, sin retardo mental ni diagnóstico de psicosis. Este inventario tiene una duración variable de aplicación entre 50 y 70 minutos. El Inventario se desarrolló en base a los cuestionarios Liverpool de Baker, et al. (1991), el ESI-55 de Vickrey, et al. (1992), el QOLIE-89 de Vickrey, et al. (1993) y el Inventario de Soporte Social (SOS) de Flaherty, Gaviria y Pathak (1988). El Inventario definitivo consta de 72 ítems en un formato tipo likert divididos en ocho dimensiones: 1. Estado de salud y realización de actividades diarias, consta de 10 ítems y un alfa de Cronbach de 0,69; 2. Efectos adversos de los medicamentos, consta de 20 ítems y un alfa de Cronbach de 0,82 ; 3 . Impacto de la epilepsia, consta de 9 ítems y un alfa de Cronbach de 0,$79 ; 4$. Apoyo Social, consta de 4 ítems y un alfa de Cronbach de 0,70; 5. Satisfacción, consta de 11 ítems y un alfa de Cronbach de 0,79; 6. Control, consta de 7 ítems y un alfa de Cronbach de 0,$75 ; 7$. Evaluación de sentimientos, consta de 8 ítems y un alfa de Cronbach de 0,73 ; y 8 . Calidad de vida global, consta de 2 ítems y un alfa de Cronbach de 0,72 . El coeficiente Alfa general del INCAVI-72 fue de 0,93. Por último, el ítems 72 no tiene una puntuación asignada ya que pretende que el sujeto enumere las dimensiones por orden de importancia de acuerdo a como ellas afectan su propia calidad de vida. En la Tabla 2 se presenta la estandarización de los puntajes de cada una de las subescalas y el puntaje total de la prueba a través de los deciles, esta estandarización a través de los deciles permitirá ubicar la calidad de vida de los sujetos en una escala de 1 a 9 . A mayor puntuación se considera una mejor calidad de vida.

\section{Procedimiento}

Previo permiso del Instituto Neurológico de Antioquia de la ciudad de Medellín, Colombia y disponibilidad a la base de datos, se contactaron a los pacientes diagnosticados con epilepsia. Los pacientes que acudían a consulta externa y que eran remitidos por el neurólogo para participar en el estudio fueron informados acerca de los objetivos de la investigación, del secreto ético de la información, que podrían retirarse del estudio cuando ellos lo consideraran y que no recibirían remuneración económica. Una vez brindada la información, se solicitó a éstos el consentimiento para participar en la investigación y una vez obtenido el consentimiento informado, cada paciente autodiligenció la ficha de datos sociodemográficos y el instrumento INCAVI-72 con la supervisión y colaboración del entrevistador cuando éstos la requerían. La aplicación tuvo una duración aproximada de una hora. 
Tabla 2. Puntajes Estandarizados de las Subescalas y Puntaje Total del INCAVI-72 (Berrío, et al., 1999, 2002)

\begin{tabular}{|c|c|c|c|c|c|c|c|c|c|c|c|}
\hline & Decil & $\begin{array}{c}\text { Subtotal } \\
\text { Saludy } \\
\text { Actividad } \\
\text { es Diarias }\end{array}$ & $\begin{array}{c}\text { Subtotal } \\
\text { Efectos } \\
\text { Adversos } \\
\text { de los } \\
\text { Medica- } \\
\text { mentos }\end{array}$ & $\begin{array}{l}\text { Subtotal } \\
\text { Impacto } \\
\text { de la } \\
\text { Epilepsia }\end{array}$ & $\begin{array}{c}\text { Subtotal } \\
\text { Apoyo } \\
\text { Social }\end{array}$ & $\begin{array}{c}\text { Subtotal } \\
\text { Satisfac- } \\
\quad \text { ción }\end{array}$ & $\begin{array}{r}\text { Subtotal } \\
\text { Control }\end{array}$ & $\begin{array}{l}\text { Subtotal } \\
\text { Evalua- } \\
\text { ción de } \\
\text { Senti- } \\
\text { miento }\end{array}$ & $\begin{array}{c}\text { Subtotal } \\
\text { Calidad } \\
\text { de Vida } \\
\text { Global }\end{array}$ & $\begin{array}{c}\text { Puntaje } \\
\text { Total } \\
\text { Prueba }\end{array}$ & Decil \\
\hline Media & & 29.23 & 63.84 & 29.35 & 13.22 & 30.84 & 25.75 & 27.55 & 10.07 & 231.28 & \\
\hline DT & & 5.40 & 14.27 & 8.24 & 3.15 & 7.44 & 5.10 & 5.33 & 1.93 & 36.48 & \\
\hline Bajo & 1 & 22.00 & 43.20 & 17.00 & 8.00 & 20.00 & 18.00 & 19.00 & 7.00 & 172.00 & 1 \\
\hline Bajo & 2 & 24.00 & 53.00 & 21.00 & 10.00 & 24.00 & 21.00 & 23.00 & 8.00 & 199.40 & 2 \\
\hline Bajo & 3 & 26.00 & 57.00 & 25.00 & 12.00 & 27.00 & 24.00 & 25.00 & 9.00 & 209.30 & 3 \\
\hline Medio & 4 & 27.00 & 62.00 & 28.00 & 13.00 & 29.00 & 26.00 & 27.00 & 10.00 & 228.60 & 4 \\
\hline Medio & 5 & 29.00 & 67.50 & 32.00 & 14.00 & 31.00 & 27.00 & 28.00 & 10.00 & 236.50 & 5 \\
\hline Medio & 6 & 32.00 & 69.60 & 33.00 & 15.00 & 34.00 & 28.00 & 30.00 & 11.00 & 246.00 & 6 \\
\hline Alto & 7 & 33.00 & 71.00 & 35.00 & 16.00 & 36.00 & 29.00 & 31.00 & 11.00 & 254.00 & 7 \\
\hline Alto & 8 & 34.20 & 77.00 & 37.00 & 16.00 & 38.00 & 30.00 & 32.00 & 12.00 & 260.60 & 8 \\
\hline Alto & 9 & 36.00 & 80.90 & 39.00 & 16.00 & 40.00 & 32.00 & 34.00 & 12.00 & 279.30 & 9 \\
\hline
\end{tabular}

\section{RESULTADOS}

\section{Análisis descriptivo de las variables clínicas}

A nivel de variables clínicas se encontró en los pacientes con epilepsia un tiempo de evolución de la enfermedad entre 1 y 14 años $(58 \%)$ y entre 15 y 50 años $(42 \%)$ y la media de años de evolución de la enfermedad fue de $15,8(D T=11,7)$. En cuanto al tipo de diagnóstico epiléptico hubo gran predominio (40\%) en el diagnóstico de epilepsia y síndromes epilépticos relacionados con localizaciones focales y parciales, seguido de un $36,6 \%$ donde la muestra presentó una incidencia variable en los diferentes diagnósticos: epilepsia convulsiva generalizada $(13,3 \%)$, epilepsia no específica (10\%) y otras epilepsias $(13,3 \%)$. De acuerdo al foco epileptogénico el 30\% de la muestra tuvieron localizaciones inespecíficas, seguido de un $21,7 \%$ donde la ubicación estuvo comprendida en el área temporal-izquierda y el $48,3 \%$ restante presentó localizaciones de variada incidencia. En relación al tratamiento farmacológico el $40 \%$ de la muestra se encontró medicada con carbamazepina y un $23,3 \%$ con ácido valpróico, el resto de la muestra empleó diferentes fármacos. (Véase la Tabla 3).

\section{Análisis descriptivo de las dimensiones del instrumento INCAVI-72}

Se observan en la Tabla 4, las diferentes medidas de tendencia central como lo son la media, la moda, la desviación típica (DT), los puntajes mínimos y máximos, y los alfa de Cronbach de cada una de las dimensiones del instrumento psicométrico INCAVI-72 que tuvo un índice de fiabilidad global de 0,70.

Según los puntajes estandarizados de las subescala y puntaje total del INCAVI-72 desarrollado por Berrío, et al. $(1999 ; 2002)$ como se observa en la Tabla 2, se analiza la CVRS de los pacientes de este estudio.

A nivel general se evidencia que los pacientes del estudio tuvieron puntuaciones medias en las dimensiones de Estado de salud $y$ actividades diarias, Satisfacción, Impacto de la epilepsia, Calidad de vida Global, Efectos adversos de los medicamentos y Apoyo Social del INCAVI-72, y se encontraron además puntuaciones bajas en las dimensiones de Evaluación de sentimientos y Control. Indicando una CVRS consistente a su cuadro clínico, es decir, entre el límite de aceptable a desfavorable. 
Tabla 3.Características de las variables clínicas

\begin{tabular}{llr}
\hline & & Frecuencia (\%) \\
\hline Tipo de Diagnóstico & Epilepsia y síntomas epilépticos sintomáticos relacionados con & \\
& localizaciones focales y parciales & $24(40 \%)$ \\
& Epilepsia convulsiva generalizada & $8(13,3 \%)$ \\
& Otras epilepsias & $8(13,3 \%)$ \\
& Epilepsia no específica & $6(10 \%)$ \\
& Epilepsias y síndromes idiopáticos generalizados & $4(6,7 \%)$ \\
& Síndromes epilépticos específicos & $4(6,7 \%)$ \\
& Otras epilepsias y síndromes generalizados & $3(5 \%)$ \\
& Epilepsia controlada & $1(1,7 \%)$ \\
& Otras convulsiones no específicas & $1(1,7 \%)$ \\
& Otras convulsiones & $1(1,7 \%)$ \\
\hline Foco Epileptogénico & Frontotemporal izquierdo & $2(3,3 \%)$ \\
& Frontotemporal derecho & $1(1,7 \%)$ \\
& Frontal izquierdo & $6(10 \%)$ \\
& Frontal derecho & $7(11,7 \%)$ \\
& Temporal derecho & $6(10 \%)$ \\
& Temporal izquierdo & $13(21,7 \%)$ \\
& Frototemporal bilatera & $11(1,7 \%)$ \\
& Temporal bilateral & $2(3,3 \%)$ \\
& Parietal derecho & $1(1,7 \%)$ \\
& Temporoparietal izquierdo & $1(1,7 \%)$ \\
& Occipital derechol (1,7\%) & \\
Rolandia & $1(1,7 \%)$ \\
Inespecífico & $18(30 \%)$ \\
\hline Carbamazepina & $24(40 \%)$ \\
& Ácido Valpróico & $14(23,3 \%)$ \\
& Fenitoina & $10(16,7 \%)$ \\
& Fenobarbital & $8(13,3 \%)$ \\
& Lacmital & $2(3,3 \%)$ \\
& Urbadan & $1(1,7 \%)$ \\
& Topiramato & $1(1,7 \%)$ \\
\hline & &
\end{tabular}

Tabla 4. Estadísticas descriptivas de cada una de las dimensiones del instrumento de Calidad de Vida Relacionada con la Salud INCAVI-72

\begin{tabular}{lcccccc}
\hline & Media & Moda & DT & Mínimo & Máximo & Alfa \\
\hline INCAVI-72 & & & & & & \\
1. Estado de salud y actividades diarias & 28,0 & 22 & 6,3 & 16 & 40 & 0,64 \\
2. Efectos de los medicamentos & 64,5 & 86 & 17,4 & 19 & 86 & 0,82 \\
3. Impacto de la epilepsia 29,1298,011420,61 & & & & & & \\
4. Apoyo Social & 13,7 & 16 & 3,1 & 4 & 16 & 0,71 \\
5. Satisfacción & 32,9 & 32 & 7,2 & 18 & 49 & 0,66 \\
6. Control & 24,7 & 20 & 5,5 & 14 & 36 & 0,65 \\
7. Evaluación de sentimientos & 26,7 & 30 & 6,3 & 8 & 36 & 0,63 \\
8. Calidad de vida Global & 9,9 & 10 & 2,0 & 5 & 14 & 0,69 \\
\hline
\end{tabular}




\section{DISCUSIÓN}

En este estudio participaron 60 pacientes con diagnóstico de epilepsia, siendo la mayoría adultos jóvenes de ambos géneros (21 a 39 años), aunque hubo mayor predomino del género femenino, laboralmente activos, solteros, con un tiempo promedio de evolución de la enfermedad de 15,8 años y de estrato socioeconómico medio-bajo de la ciudad de Medellín, Colombia. El coeficiente de consistencia interna alfa de Cronbach del INCAVI-72 fue de 0,70 en este estudio.

Es de considerar que en la calidad de vida del paciente epiléptico intervienen numerosos factores que producen un deterioro progresivo en sus vidas independientemente de la etapa del ciclo vital en que se encuentren estas personas. Entre los factores más relevantes se encuentran: las crisis, algunos relacionados con la enfermedad y su tratamiento y otros con una relación menos directa como la percepción de la enfermedad por parte del sujeto y la sociedad, la presencia o ausencia de psicopatología asociada y la comorbilidad (Gil-Nagel y Marinas, 2006).

En este estudio los pacientes con diagnóstico de epilepsia tuvieron una CVRS a nivel general dentro de los límites normales con una ligera tendencia hacia puntuaciones bajas. Es decir, estos pacientes a nivel de bienestar físico $\mathrm{y}$ de funcionamiento cognoscitivo pueden desempeñarse en las diferentes tareas en las que participan sin dificultades significativas (Estado de salud y actividades diarias), tienen cierto bienestar consigo mismo y con aspectos de su vida familiar, social, laboral y sexual (Satisfacción), y tuvieron las posibilidades de contar con ayuda emocional, económica y de atención en el momento que lo requirieron de manera oportuna a su sentir (Apoyo Social). Además, hubo en ellos una afectación normal o la esperada respecto a los tratamientos para el manejo de la epilepsia en relación a las manifestaciones físicas, cognoscitivas, emocionales y comportamentales que se dan como resultado de la ingesta de los medicamentos anticonvulsivantes (Efectos adversos de los medicamentos), a nivel general la epilepsia y su tratamiento no le generaron a estos pacientes alteraciones significativas en los diferentes aspectos relaciona- dos con su vida diaria (Impacto de la epilepsia). Por otro lado, en estos pacientes se evidencia niveles de riesgo clínicamente significativos por la presencia de emociones negativas (Evaluación de sentimientos), y de cierta inhabilidad en la percepción de dominio frente a las situaciones de su vida diaria y del futuro (Control). Aunque los estados emocionales de los pacientes de este estudio son desfavorables, es de considerar que la percepción general de CVRS es adecuada en relación con la satisfacción de sus necesidades básicas, en el bienestar con la vida y en sus proyecciones personales (Calidad de vida Global).

Respeto a los resultados de la validación original de la investigación de Berrío, et al. (1999) que se desarrolló en la ciudad de Bogotá, Colombia con una muestra de 100 personas adultas con diagnóstico de epilepsia se encontraron resultados significativamente similares en todas las dimensiones del INCAVI-72 con nuestro estudio. En la dimensión de Estado de salud y actividades diarias en el estudio de Berrío, et al. (1999) se encontró una puntuación media de 29,2 y en el nuestro de 28,0; en la dimensión de Efectos de los medicamentos en el estudio de Berrío, et al. (1999) se encontró una puntuación media de 63,8 y en el nuestro de 64,5; en la dimensión de Impacto de la epilepsia en el estudio de Berrío, et al. (1999) se encontró una puntuación media de 29,3 y en el nuestro de 29,1; en la dimensión de Apoyo Social en el estudio de Berrío, et al. (1999) se encontró una puntuación media de 13,2 y en el nuestro de 13,7; en la dimensión de Satisfacción en el estudio de Berrío, et al. (1999) se encontró una puntuación media de 30,8 y en el nuestro de 32,9; en la dimensión de Control en el estudio de Berrío, et al. (1999) se encontró una puntuación media de 25,7 y en el nuestro de 24,7; en la dimensión de Evaluación de sentimientos en el estudio de Berrío, et al. (1999) se encontró una puntuación media de 27,5 y en el nuestro de 26,7; y en la dimensión de Calidad de vida Global en el estudio de Berrío, et al. (1999) se encontró una puntuación media de 10,0 y en el nuestro de 9,9.

Cuando se ha analizado la calidad de vida numerosos estudios han identificado factores importantes como emociones negativas (depre- 
sión y ansiedad), alteraciones cognitivas, estigma social y apoyo social, la situación laboral y preocupaciones por los efectos secundarios a nivel físico y mental de la medicación antiepiléptica que influyen en la calidad de vida de esta población (Cramer, et al., 2003; OliverosCid, et al., 2005; Ríos y Hernández, 2005; Mosaku, et al., 2006; Gil-Nagel y Marinas, 2006; Huang, et al., 2007; Guekht, et al., 2007). Otros estudios han planteado que hasta un $44 \%$ de los pacientes pueden estar preocupados por los posibles efectos adversos de su tratamiento (Baker, Jacoby, Buck, Stalgis y Monnet, 1997). Por otro lado, Suurmeijer, Reuvekamp y Aldenkamp (2001), en una muestra de 210 pacientes con epilepsia encontraron que las variables médicas y clínicas (tipo y gravedad de la crisis, percepción del estado de salud) influyeron únicamente en un $15 \%$ sobre la CVRS, mientras que las variables psicosociales, especialmente las emociones negativas influyeron en más del $30 \%$.

Los anteriores datos son parcialmente similares a nuestro estudio, ya que, por un lado, factores relacionados con el tratamiento y la epilepsia, las funciones físicas y la satisfacción con los diferentes roles que son desempeñados en la vida no fueron afectados por las manifestaciones etiopatologicas de la enfermedad, más sin embargo, las emociones negativas y el control personal siguen siendo factores de marcada relevancia negativa en estos pacientes igual que en los otros estudios.

Según Barra (2003) los estados emocionales pueden influir en la salud mediante efectos directos en el funcionamiento fisiológico, en el reconocimiento de síntomas en la búsqueda de atención médica como en estrategias de regulación emocional la cual está mediada por el control personal. La creencia de control y la sensación de autoeficacia en relación a la enfermedad y su tratamiento son generalmente adaptativos y hasta puede prolongar la vida de quien padece una determinada enfermedad. En diferentes estudios con enfermos crónicos se han reportado que quienes creen tener el control sobre su enfermedad se ajustan mejor a su condición física y psicosocial que aquellos que carecen de esta creencia (Sanjuán y Magallares, 2006; Talarn, et al., 2006; Taylor, 2007).
Es de resaltar que los estudios de CVRS en personas adultas con epilepsia son muy escasos, especialmente en América Latina y prácticamente inexistentes a nivel de publicaciones en Colombia. Sería importante, dada la alta prevalencia de esta enfermedad crónica en Iberoamérica que se desarrollen investigaciones que evalúen aspectos asociados con el bienestar psicológico y el impacto de emociones negativas y positivas en las personas que padecen esta enfermedad. De otro lado, sería interesante realizar estudios que analicen las relaciones entre crisis epilépticas y la CVRS y el papel que puedan tener los estados depresivos en su evolución como la personalidad. El análisis de la calidad de vida en la epilepsia permite establecer una valoración global de la valoración clínica y social del enfermo, y con ello evaluar los resultados del tratamiento de una forma más realista (Gil-Nagel y Marinas, 2006).

\section{REFERENCIAS}

Adams, R.D. (1997). Epilepsy and other seizure disorders. En R.D. Adams, M. Victor y A.H. Ropper (Eds.), Principles of Neurology (pp. 313-341). New York: McGraw-Hill.

Baker, A., Jacoby, A., Buck, D., Stalgis, C., y Monnet, D. (1997). Quality of life of people with epilepsy: a European study. Epilepsia, 38, 353-362.

Baker, G.A., Smith, D.F., Dewey, M., Morrow, J., Crawford, P., y Chadwick, D.W. (1991). The development of a seizure severity scale as an outcome measure in epilepsy. Epilepsy Research, 8, 245-251.

Barra, E. (2003). Influencia del estado emocional en la salud física. Terapia Psicológica, 21, 55-60.

Bender, J.E., Álvarez, E., y Rodríguez, L. (2006). Enfermedad cerebrovascular y epilepsia. Análisis casuístico del CIREN. Revista Mexicana de Neurociencia, 7, 325-329.

Bender, J.E., Morales, L., Paz, L., y Prida, M. (2005). Bioética y epilepsia refractaria. Revista Mexicana de Neurociencia, 6, 153-161.

Berrío, G.M. (2002). Diseño, construcción y validación de un Inventario para evaluar Calidad de Vida en adultos con Epilepsia. Acta Colombiana de Psicología, 7, 7-19.

Berrío, G.M., Abaunza, L., Herrera, P., y Salas, D.J. (1999). Manual Inventario para la evaluación de la Calidad de Vida en adultos con epilepsia INCAVI-72. Manuscrito no publicado, Bogotá D.C., Colombia. 
Brannon, L., y Feist, J. (2001). Psicología de la Salud. Madrid: Paraninfo.

Burneo, J.G., Tellez-Zenteno, J., y Wiebe, S. (2005). Understanding the burden of epilepsy in Latin America: a systematic review of its prevalence and incidence. Epilepsy Research, 66, 63-74.

Carrizosa, J. (2007). Prevalencia, incidencia y brecha terapéutica en la epilepsia. Iatreia, 20, 282-296.

Commission on Classification and Terminology of the International League Against Epilepsy - ILAE- . (1989). Proposal for revised classification of epilepsies and epileptic syndromes. Epilepsia 30, 389-399.

Cramer, J.A., Blum, D., Reed, M., y Fanning, K. (2003). The influence of comorbid depression on quality of life for people with epilepsy. Epilepsy Behavior, 4, 515-521.

Cramer, J.A., Perrine, K., Devinsky, O., y Meador, K. (1996). A brief questionnaire to screen for quality of life in epilepsy: the QOLIE-10. Epilepsia, 37, 577-582.

Días, J.J. (2002). Epilepsia. Recuperado el 10 de noviembre de 2007, del sitio Web de Fisterra: Atención Primaria en la red: http://www.fisterra.com/guias2/PDF/Epilepsia.pdf

Dodrill, C.B., Batzel, L.W., Queisser, H.R., y Temkin, N.R. (1980). An objective method for the assessment of psychological and social problems among epileptics. Epilepsia, 21, 123-35.

Flaherty, J.A., Gaviria, F., y Pathak, D. (1988). Developing Instruments for cultural research. Journal Nervous and Mental Disease, 176, 257-263.

Forsgren, L. (1997). Epidemiology in Epilepsy. Epilepsy primarily affects small children and the elderly. Lakartidningen, 94, 1899-1902.

García-Albea, E. (1999). Historia de la Epilepsia. Barcelona: Masson.

Gil-Nagel, A., y Marinas, A. (2006). Calidad de vida en la epilepsia. En P. Martínez-Martín (Ed.), Calidad de vida en neurología (pp. 251-263). Barcelona: ARS Médica.

Giovagnoli, A.R., Meneses, R.F., y Da Silva, A.M. (2006). The contribution of spirituality to quality of life in focal epilepsy. Epilepsy Behavior, 9, 133-139.

Guekht, A.B., Mitrokhina, T.V., Lebedeva, A.V., Dzugaeva, F.K., Milchakova, L.E., Lokshina, O.B., et al. (2007). Factors influencing on quality of life in people with epilepsy. Seizure, 16, 128-133.

Herranz, J.L. (1999). Problemática social y calidad de vida del niño epiléptico. Procedimientos para objetivarlas y para mejorarlas. Boletín de Pediatría, 39, 28-33.

Huang, R.Y., Xie, J.S., Li, X.B., y Wei, S.G. (2007). Quality of life of epilepsy patients in Zhuang populations in Guangxi Guixi area. Zhongguo Yi Xue Ke Xue Yuan Xue Bao, 29, 103-106.

Jiménez, M.D. (2004). Continua neurológica: Epilepsia. Barcelona: ARS Medica.
Laccheo, I., Ablah, E., Heinrichs, R., Sadler, T., Baade, L., y Liow, K. (2008). Assessment of quality of life among the elderly with epilepsy. Epilepsy Behavior, 12, 257261.

Morales, L.M. (2002). Cirugía de la epilepsia: consideraciones éticas acerca del consentimiento informado y la calidad de vida. Revista Mexicana de Neurociencias, 3, 109-115.

Mosaku, K.S., Fatove, F.O., Komolafe, M., Lawal, M., y Ola, B.A. (2006). Quality of life and associated factors among adults with epilepsy in Nigeria. International Journal Psychiatric Medicine, 36, 469-481.

Oblitas, L. (2006). Psicología de la Salud y calidad de vida. México: Thompson Learning.

Oliveros, A. (1997). Aplicación de la escala de calidad de vida del adulto con epilepsia (FEGEA). Revista de Neurología, 25, 422-428.

Oliveros-Cid, A., Oliveros-Juste, A., y Cid, M.A. (2005). Calidad de vida y discapacidad del anciano epiléptico. En F.J. Vadillo-Olmo y J.A. Mauri-Llerda (Eds.), Crisis y epilepsia en el anciano (pp.169-196). Barcelona: Viguera Editores.

Padilla, G. (2005). Calidad de vida: panorámica de investigaciones clínicas. Revista Colombiana de Psicología, $13,80-88$.

Ríos, J.R., y Hernández, M. (2005). Prevalencia de depresión en pacientes con epilepsia y su calidad de vida. Revista de Sanidad Militar, 59, 311-317.

Sanjuán, P., y Magallares, A. (2006). Estilo atributivo negativo, sucesos vitales y sintomatología depresiva. Revista de Psicopatología y Psicología Clínica, 11, 9198.

Schwartzmann, L. (2003). Calidad de vida relacionada con la salud: aspectos conceptuales. Ciencia y Enfermería, 2, 9-21.

Simón, M.A. (1999). Tratado de psicología de la salud. Madrid: Biblioteca Nueva.

Suurmeijer, T., Reuvekamp, M.F., y Aldenkamp, B.P. (2001). Social Functioning, psychological fuctioning, and quality of life in epilepsy. Epilepsia, 42, 11601168.

Takeuchi, Y., y Guevara, J.G. (1999). Prevalencia de las enfermedades neurológicas en el Valle del Cauca. Estudio Neuroepidemiológico Nacional (EPINEURO). Colombia Médica, 30, 74-81.

Talarn, A., Navarro, N., Rossell, L., y Rigat, A. (2006). Propuesta de especificadores diagnósticos vinculados al estrés y el trauma: una aportación a la nosología psicopatológica. Revista de Psicopatología y Psicología Clínica, 11, 107-114.

Taylor, S. (2007). Psicología de la Salud (6 ${ }^{\mathrm{a}}$ ed.). México: McGraw-Hill.

Torres, X., Arroyo, S., Araya, S., y De Pablo, J. (1999). The Spanish Version of the Quality-of-Life in Epilep- 
sy Inventory (QOLIE-31): Translation, Validity, and Reliability. Epilepsia, 40, 1299-1304.

Vélez, A., y Eslava-Cobos, J. (2006). Epilepsy in Colombia: epidemiologic profile and classification of epileptic seizures and syndromes. Epilepsia, 47, 193-201.

Vickrey, B.G., Hays, R.D., Graber, J., Rausch, R., Engel, J., y Brook, R.H. (1992). A health-related quality of life instrument for patients evaluated for epilepsy surgery. Medical Care, 30, 299-319.

Vickrey, B.G., Perrine, K.R., Hays, R.D., Hermann, B.P., Cramer, J.A., Gordon, J., et al. (1993). Scoring Manual for the QOLIE-89, version 1.0. Santa Mónica, California: RAND. 